\title{
Análisis de los factores de productividad, desperdicio y confiabilidad de los equipos, al implementar TPM en una empresa del sector automotriz
}

\section{Analysis of the factors of productivity, waste and reliability of the equipment, when implementing TPM in a company of the automotive sector}

\author{
LANDEROS-CORREA, Carmen †*, CHIHUAQUE-ALCANTAR, Jesús, MELESIO-MORENO, Ma. \\ Guadalupe y GALVÁN-GARCÍA, María Isabel
}

Universidad Politécnica de Guanajuato, Ingeniería Automotriz

ID $1^{\mathrm{er}}$ Autor: Carmen, Landeros-Correa / ORC ID: 0000-0002-7896-0613, CVU CONACYT ID: 328162

ID $1^{\text {er }}$ Coautor: Jesús, Chihuaque-Alcantar / ORC ID: 0000-0002-6718-6909, CVU CONACYT ID: 48887

ID $2^{\text {do }}$ Coautor: Ma. Guadalupe, Melesio-Moreno / ORC ID: 0000-0002-1346-1186

ID $3^{\text {er }}$ Coautor: María Isabel, Galván-García / ORC ID: 0000-0003-0086-6739

DOI: $10.35429 /$ JIE.2019.10.3.36.45

Recibido 19 de Octubre, 2019, Aceptado, 04 de Diciembre, 2019

\begin{abstract}
Resumen
El mantenimiento productivo total (TPM), es una de las prácticas más usadas por las empresas automotrices, para incrementar su productividad, motivo por el cual se realiza esta investigación en una empresa automotriz validando este supuesto, a través de la implementación del TPM en una línea productiva y determinando mediante un diseño de experimento, si el TPM afecta o no de manera significativa a los indicadores de eficiencia, desperdicio, confiabilidad de los equipos, debido a que estos indicadores son importantes para la productividad de la empresa. Con dicho análisis se logró demostrar, para este caso en particular, que la herramienta de TPM contribuye en la mejora de dos de los indicadores. Aunque esta conclusión aplica para la empresa y la línea en la cual fue aplicado dicho análisis, en las condiciones en las cuales fue evaluada la herramienta; para que ésta sea más general, se tiene que evaluar en otras empresas y comparar el comportamiento de dicha herramienta en cada una de ellas.
\end{abstract}

TPM, Mejora, Diseño de experimentos

\begin{abstract}
Total productive maintenance (TPM), is one of the practices most used by automotive companies, to increase their productivity, which is why this research is carried out in an automotive company validating this assumption, with the implementation of the TPM in a productive line and determining by means of an experiment design, if the TPM does not significantly affect the indicators of efficiency, waste, reliability of the equipment, because these indicators are important for the productivity of the company. With this analysis it was possible to demonstrate, for this particular case, that the MPR tool does help improve two of the indicators, although this conclusion only applies to this company and the line in which said analysis was applied, under the conditions in which was evaluated the tool, so that this conclusion is more general, it has to be evaluated in other companies and to be able to make the comparison of the behavior in each of them.
\end{abstract}

TPM, Improvement, Experiment design

Citación: LANDEROS-CORREA, Carmen, CHIHUAQUE-ALCANTAR, Jesús, MELESIO-MORENO, Ma. Guadalupe y GALVÁN-GARCÍA, María Isabel. Análisis de los factores de productividad, desperdicio y confiabilidad de los equipos, al implementar TPM en una empresa del sector automotriz. Revista de Ingeniería Industrial. 2019 3-10: 36-45

\footnotetext{
* Correspondencia del Autor (Correo electrónico: clanderos@ upgto.edu.mx)

$\dagger$ Investigadora contribuyendo como primer autor.
} 


\section{Introducción}

En el documento denominado "Conociendo la industria automotriz" se presentan los indicadores más importantes de una de las industrias con mayor relevancia en el país.

La industria automotriz en México aportó $3.7 \%$ del PIB nacional y $20.2 \%$ del PIB manufacturero en 2017. México es el $4^{\circ}$ exportador mundial de productos de la industria automotriz. (INEGI, AMIA, 2018, párr. 5)

El progreso industrial no significa inversiones en nuevas máquinas o tecnologías, sino que es primordial utilizar lo existente de forma eficaz, donde se establezca un servicio de producción eficiente, seguro y económico con los equipos industriales. (Pinto, 2010)

De la importancia que ésta industria tiene en el país, se deriva la inminente necesidad de aplicar herramientas que contribuyan a la productividad de la misma, siendo el TPM una de estas herramientas que ayuda a contribuir en este indicador.

Es así que, el concepto de TPM fue introducido en 1971, por los japoneses quienes lo desarrollaron después de la segunda guerra mundial donde llegaron a la conclusión que debían mejorar la calidad de sus productos; es por esta razón, que se basaron en las técnicas de gestión y fabricación provenientes de EEUU y las adaptaron a sus propias circunstancias. (Partida, 2012).

El TPM es altamente eficaz en aquellas empresas que cuentan con operaciones automáticas y secuenciales (empresas intensivas en el uso de maquinaria), y no requieren grandes inversiones económicas para lograr el aprovechamiento de las instalaciones existentes. (García, 2011)

En el documento "Importancia del mantenimiento productivo total en la automatización de procesos" menciona que [...] El TPM contiene los siguientes puntos importantes

1. Tiene como objetivo el uso más eficiente del equipo (mejora de la eficiencia global).
2. Establece un sistema de mantenimiento productivo en toda la empresa para la vida entera del equipo. Incluye prevención del mantenimiento relacionado con mejoras.

3. Promociona y lleva a cabo mantenimiento productivo a través de la gestión de la motivación basada en actividades autónomas en grupos pequeños. (Calvo, Lago, 2004, p, 2)

El objetivo de esta investigación consiste en analizar los factores que impactan al implementar la metodología de TPM (Mantenimiento Productivo Total) mediante una investigación de tipo cuantitativa con diseño de experimentos que nos permita comparar el efecto de dicho experimento.

En los últimos años, la aplicación del diseño experimental ha florecido notablemente debido al reconocimiento que los experimentos diseñados son esenciales para la mejora de la calidad de productos y servicios. (Napolitano, sf) Se entiende el "Diseño de Experimentos como una metodología para aplicar sistemáticamente la estadística al proceso de experimentación". Más técnicamente, consiste en realizar una serie de pruebas en las que se inducen cambios deliberados en las variables de un proceso de manera que es posible observar e identificar las causas de los cambios en la respuesta de salida elegida. (Tanco, Viles, Pozuela. 2009)

Con la implementación del TPM en la línea 3 se quiere probar si existe un efecto o no, en los indicadores de productividad, desperdicio y confiabilidad, para lo cual usaremos un diseño de experimentos completamente al azar.

El diseño completamente al azar es el más sencillo de los diseños de experimentos que tratan de comparar dos o más tratamientos, puesto que solo considera dos fuentes de variabilidad: los tratamientos y el error aleatorio. (Yepes, 2013, párr. 1)

Se utiliza el análisis de varianza (ANOVA) para comprobar si existen diferencias en las medias. Fundamentalmente este análisis consiste en separar la contribución de cada fuente de variación en la variación total observada. (Yepes, 2013, párr. 6). 


\section{Metodología}

El estudio es de corte cuantitativo, ya que se usará para el análisis las magnitudes numéricas de los indicadores, productividad, desperdicio y confiabilidad.

Así también, la investigación es experimental debido a que se manipula la variable de implementación de TPM, que para ello usaremos un diseño completamente al azar.

El estudio se aplicó en la línea 3 de una empresa dedicada a realizar pistones y módulos de potencia para la industria automotriz.

Para ello, se usará el cálculo de la productividad en la línea. En general, la productividad se mide por el coeficiente formado por los resultados logrados y los recursos empleados. Los resultados logrados pueden medirse en unidades producidas, en piezas vendidas o en utilidades, mientras que los recursos empleados pueden cuantificarse por número de trabajadores, tiempo total empleado, horas máquina, etc.

Productividad "Es el resultado final de un complejo proceso social, compuesto por ciencia, investigación y desarrollo, educación, tecnología, dirección de empresa, medios de producción y organización de trabajadores." (Kurosawa, 1983)

Productividad: mejoramiento continuo del sistema Más que producir rápido, se trata de producir mejor Productividad $=$ Eficiencia $\mathrm{x}$ eficacia

$\frac{\text { Unidaeds producidas }}{\text { Tiempo total }}=\frac{\text { Tiempo útil }}{\text { Tiempo total }} x \frac{\text { Unidades producidas }}{\text { Tiempo útil }}$

\section{Eficiencia $=\mathbf{5 0 \%}$}

$50 \%$ del tiempo se desperdicia en:

- Programación

- Paros no programados

- Desbalanceo de capacidades

- Mantenimiento y reparaciones

Figura 1 La productividad y sus componentes Fuente: Pulido, 2010

En la figura 1, se muestran los componentes de la productividad y se ejemplifica la definición de eficacia y eficiencia midiendo los recursos empleados a través del tiempo total y los resultados mediante la cantidad de productos generados en buenas condiciones.
Según una encuesta aplicada en los sectores metalmecánico, calzado, muebles, textil y confección en México (Giral et al., 1998), la eficiencia promedio detectada fue de $50 \%$, es decir, en estos sectores se desperdiciaba la mitad del tiempo, en promedio, en aspectos inherentes a fallas de planeación y organización de la producción, principalmente. (Gutiérrez, 2010)

Existen muchos métodos para la medición de la productividad dependiendo de las variables que podemos calcular en planta.

Algunos métodos utilizados para la medición de productividad son los que se muestra en la tabla 1.

\begin{tabular}{|l|l|l|}
\hline \multicolumn{1}{|c|}{ Método } & \multicolumn{1}{c|}{$\begin{array}{c}\text { Nivel de } \\
\text { aplicación }\end{array}$} \\
\hline BLS & $\begin{array}{l}\text { Nacional } \\
\text { Rama } \\
\text { Empresa }\end{array}$ & $P=\frac{1}{\text { índice de producto }}$ \\
\hline $\begin{array}{l}\text { Earl Burch } \\
\text { Jr. }\end{array}$ & $\begin{array}{l}\text { Rama } \\
\text { Empresa }\end{array}$ & $P T=\frac{\text { Valor bruto de la producción }}{\text { MO }+ \text { CAP }+ \text { GI }+ \text { MAT }}$ \\
\hline OIT & $\begin{array}{l}\text { Nacional } \\
\text { Rama } \\
\text { Empresa }\end{array}$ & $P N=\frac{\text { producto Interno Bruto }}{\text { Población Econ. Activa }}$ \\
\hline $\begin{array}{l}\text { Enrique } \\
\text { Hernández } \\
\text { Láos }\end{array}$ & $\begin{array}{l}\text { Nacional } \\
\text { Empresa }\end{array}$ & productividad $=\frac{\text { producto obtenido }}{\text { trabajo }+ \text { capital }}$ \\
\hline
\end{tabular}

Tabla 1 Productividad y sus componentes

Fuente: Recuperado de www.geocities.com/unamos apuntes_2000/apuntes/seminariodelIPN.doc

Uno de los indicadores más utilizados en las empresas de clase mundial para medir la productividad es el OEE, ya que con este indicador se engloba la disponibilidad por el rendimiento y por la calidad.

$\mathrm{OEE}=$ Producción real/Máxima producción teórica.

OEE $=$ Tasa de Disponibilidad $\mathrm{x}$ Tasa de Rendimiento x Tasa de Calidad.

Tasa de Disponibilidad - La proporción del tiempo de producción real y el tiempo de producción planificado. Todas las detenciones planificadas e interrupciones reducirán la tasa de disponibilidad, incluyendo los tiempos de preparación, mantenimiento preventivo, averías y falta de operarios.

El único tiempo que usted puede elegir para deducirlo de la tasa de disponibilidad es la falta de pedidos. 
Tasa de Rendimiento - Pérdida de producción debido a la subutilización de la maquinaria. En otras palabras, se incurre en pérdidas cuando el equipo no está funcionando a toda velocidad. Las detenciones breves, no registradas también pueden afectar la tasa de rendimiento.

Tasa de Calidad - La cantidad de producción que tiene que ser eliminada $\mathrm{o}$ desechada. (Olofsson, 2013)

Desperdicio - Es todo aquello que no agrega valor a un producto o servicio para los clientes, perdida o despilfarro, en este contexto, es toda mal utilización de los recursos.

Si bien no es una situación deseable, las operaciones producen partes que ya no se pueden utilizar (scrap). Además, existe la necesidad de repetir éstas, una o varias veces, por la parte no se produjo al primer intento dentro de las especificaciones establecidas; a esto se le denomina retrabajo.

El desperdicio y el retrabajo ocasionan el uso ineficiente y derrochador de los recursos de las instalaciones. Debe hacerse todo para eliminar este desperdicio. Sin embargo, en tanto la planta tiene que vérselas con el desperdicio y el re-trabajo, o es posible ignorar la demanda que ejercen de tiempo de producción. (Meyer y Stephens, 2006).

Los departamentos de calidad y de producción tienen datos históricos que indican el nivel de retrabajos y desperdicio de cada operación. Para determinar la tasa de la planta, o el tiempo de procesamiento, en los cálculos debe incluirse la tasa de desperdicio y de retrabajo. Además, también es prudente agregar en esos cálculos las apartes de refacción o de remplazo que se necesita. (Meyer y Stephens, 2006).

Con los datos obtenidos se calcula el índice de desperdicio quedando como fórmula general la siguiente:

$$
I=\frac{\text { Salidas }}{(1-\% \text { de desperdicio } 1) \ldots(1-\% \text { de desperdicio } n)}
$$

Fuente: Meyer y Stephens, 2006

Con este indicador se evaluará la parte de desperdicio en nuestro análisis.

Confiabilidad.
Para tener claro el horizonte, en lo referente al cálculo; se debe conocer con certeza el concepto de confiabilidad, la cual se puede definir de la siguiente manera: Es la probabilidad de que un sistema, activo o componente lleve a cabo su función adecuadamente durante un período bajo condiciones operacionales previamente definidas y constantes. (Huerta, 2006)

Para la parte operacional en el análisis de la línea 3, se evaluarán los indicadores de MTBF y MTTR dentro de la confiabilidad de los equipos. indicadores.

Descripción de cada uno de estos

MTBF: Tiempo medio del buen funcionamiento entre paradas.

MTTR: Tiempo medio de cada parada.

Confiabilidad $=\frac{\mathrm{MTBF}}{\mathrm{MTBF}+\mathrm{MTTR}}$

Fuente: Recuperada de Gonzales Fernandez, F. J. (s.f.).

Una vez que definidos los indicadores que se utilizarán y como se usarán, se procederá al análisis.

\section{Diseño de experimento}

Existen diferentes diseños de experimentos para poder comparar dos tratamientos, como lo son: Diseño completamente al azar, diseño de bloque completamente al azar, diseño de cuadrado latino y grecolatino. Para el presente análisis, se utilizará el Diseño completamente al azar debido a que no se cuenta con algún otro factor de interés.

Para validar que existe diferencia significativa entre aplicar o no TPM; en relación a los indicadores analizados, se realizará un ANOVA de un Diseño de experimentos completamente al azar (DCA).

El objetivo del ANOVA en el DCA es probar las hipótesis de igualdad de los tratamientos con respecto a la media correspondiente da la variable de respuesta.

Ho: $\mu_{\mathrm{A}}=\mu_{\mathrm{B}}=\ldots=\mu_{\mathrm{j}}$

Ha: $\mu_{\mathrm{i}} \neq \mu_{\mathrm{j}}$ para algún $\mathrm{i} \neq \mathrm{j}$ 
Una medida de la Total, presente en las observaciones de un diseño de experimentos, es la suma de cuadrados que se calcula con la siguiente ecuación.

$S C_{T}=\sum_{i=1}^{k} \sum_{j=1}^{n i} Y_{i j}^{2}-\frac{Y_{. .}^{2}}{N}$

Fuente: Gutiérrez H, De la Vara R (2012)

Donde $\mathrm{Yij}^{2}$ es la cada uno de los valores obtenidos en los análisis elevados al cuadrado, Y.. ${ }^{2}$ es la suma total de los datos del experimento.

La suma de cuadrados de los tratamientos se calcula con la siguiente formula:

$\mathrm{SC}_{\text {TRAT }}=\sum_{\mathrm{i}=1}^{\mathrm{k}} \frac{\mathrm{Y}_{\mathrm{i}}{ }^{2}}{\mathrm{n}}-\frac{\mathrm{Y..}^{2}}{\mathrm{~N}}$

Fuente: Gutiérrez H, De la Vara R (2012).

En donde $\mathrm{Yi}^{2}{ }^{2}$ es la suma de cada tratamiento elevado al cuadrado, $\mathrm{n}$ es el número de réplicas y $\mathrm{N}$ es el número total de datos.

Para calcular el $\mathrm{SC}_{\mathrm{E}}$ que sería el cuadrado medio del error, este se calcula con la diferencia de la $\mathrm{SC}_{\mathrm{T}}-\mathrm{SC}_{\text {Trat }}$.

$\mathrm{SC}_{\mathrm{E}}=\mathrm{SC}_{\mathrm{T}}-\mathrm{SC}_{\text {Trat }}$

Fuente: Gutiérrez H, De la Vara R (2012)

\begin{tabular}{|c|c|c|c|c|c|}
\hline $\mathrm{FV}$ & $\mathrm{SC}$ & GL & $\mathrm{CM}$ & Fo & Valor-p \\
\hline Tratamientos & $\mathrm{SC}_{\text {Trat }}$ & $\mathrm{k}-1$ & $\begin{array}{l}C M_{\text {Trat }} \\
=\frac{S C_{\text {Trat }}}{k-1}\end{array}$ & $\frac{C M_{\text {Trat }}}{C M_{E}}$ & $\mathrm{P}(\mathrm{F}>\mathrm{Fo})$ \\
\hline Error & $\mathrm{SC}_{\mathrm{E}}$ & N-k & $\begin{array}{l}C M_{E} \\
=\frac{S C_{E}}{N-k}\end{array}$ & & \\
\hline Total & $\mathrm{SC}_{\mathrm{T}}$ & $\mathrm{N}-1$ & & & \\
\hline
\end{tabular}

Tabla 2 Tabla de ANOVA para DCA

Fuente: Gutiérrez H, De la Vara R (2012)

Una vez obtenido el ANOVA, se puede concluir con el valor-p. Si valor-p> a alfa se acepta Ho, por lo cual concluimos que las medias son iguales. Si valor-p $<$ a alfa se rechaza Ho, por lo cual concluimos que las medias son diferentes y aceptamos Ha.

La validez de los resultados obtenidos en cualquier análisis de varianza queda supeditado a que los supuestos del modelo se cumplan. Estos supuestos son: normalidad, varianza constante (igual varianza de los tratamientos) e independencia.
Esto es, la respuesta $(Y)$ se debe distribuir de manera normal, con la misma varianza en cada tratamiento y las mediciones deben ser independientes.

Estos supuestos sobre $\mathrm{Y}$ se traducen en supuestos sobre el término error $(\varepsilon)$.

Para comprobar cada supuesto existen pruebas analíticas y gráficas. Por su sencillez muchas veces se prefieren las pruebas gráficas. Normalidad.

Un procedimiento grafico para verificar el cumplimiento del supuesto de normalidad de los residuos consiste en graficar los residuos en papel o en la gráfica de probabilidad normal que se incluye casi en todos los paquetes estadísticos.

Los datos deben de ser normales para ello se buscará el apoyo del análisis que proporciona la gráfica de normalidad, misma que puede ser calculada en Minitab.

Se calculará cada una de las gráficas para los datos con y sin TPM.

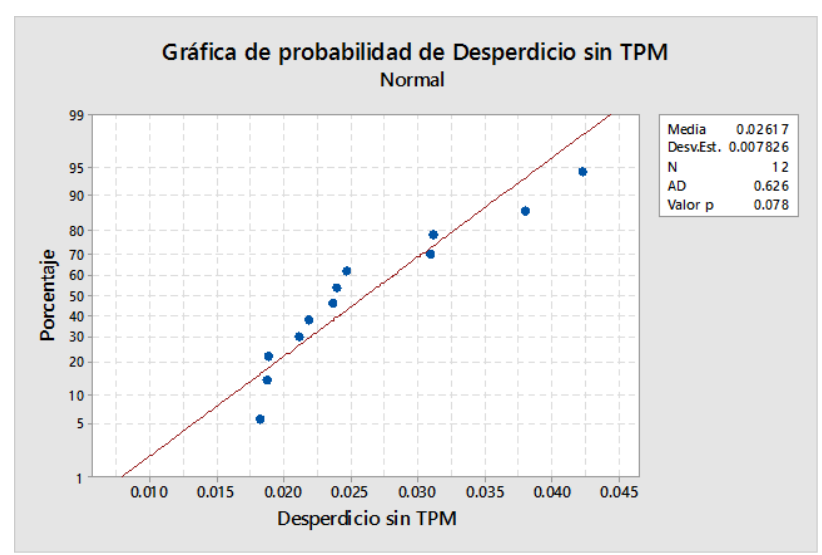

Figura 2 Representación de grafica de papel normal Fuente: Recuperado de Minitab 17

Varianza constante (igual varianza en los tratamientos).

Para este análisis, también se recurrirá al Minitab y sólo se llevará a cabo un análisis para cada indicador debido a que éste consiste en la comparación de más varianzas de sin y con TPM.

\section{Independencia}

Esto consiste en que todos los diseños de experimentos son interdependientes.

LANDEROS-CORREA, Carmen, CHIHUAQUE-ALCANTAR, Jesús, MELESIO-MORENO, Ma. Guadalupe y GALVÁN-GARCÍA, María Isabel. Análisis de los factores de productividad, desperdicio y confiabilidad de los equipos, al implementar TPM en una empresa del sector automotriz. Revista de Ingeniería Industrial. 2019 
Una vez que se probaron los supuestos, se procedió a realizar el ANOVA el cual contribuirá a una toma de decisión fundamentada estadísticamente en la existencia o no, de diferencia al aplicar el TPM para cada uno de los indicadores analizados en ésta investigación.

\section{Resultados}

Una vez analizados los supuestos que se encuentran en el Anexo y verificando que todo se cumple, se procedió a realizar el Análisis de Varianza de cada uno de los indicadores.

Los resultados en cada uno de los indicadores mencionados, fueron los siguientes:

Productividad

Hipótesis a probar

Ho: $\mu_{\operatorname{sinTPM}}=\mu_{\text {conTPM }}$

Ha: $\mu_{\operatorname{sinTPM}} \neq \mu_{\text {conTPM }}$

Para la parte de productividad se calculó para los 12 meses del año.

\begin{tabular}{|c|c|}
\hline $\begin{array}{c}\text { Productividad sin } \\
\text { TPM }\end{array}$ & $\begin{array}{c}\text { Productividad con } \\
\text { TPM }\end{array}$ \\
\hline 0.806 & 0.810 \\
\hline 0.780 & 0.816 \\
\hline 0.747 & 0.845 \\
\hline 0.749 & 0.830 \\
\hline 0.794 & 0.831 \\
\hline 0.751 & 0.823 \\
\hline 0.790 & 0.820 \\
\hline 0.780 & 0.820 \\
\hline 0.770 & 0.814 \\
\hline 0.788 & 0.828 \\
\hline 0.779 & 0.819 \\
\hline 0.779 & 0.820 \\
\hline
\end{tabular}

Tabla 3 Resultados de productividad sin y con TPM Fuente: Elaboración propia

Usando el software de Minitab 17 se obtuvo el siguiente análisis de varianza, con un alfa de 0.05 .

\begin{tabular}{|c|c|c|c|c|c|}
\hline Fuente & GL & $\begin{array}{l}\text { SC } \\
\text { Ajust. }\end{array}$ & $\begin{array}{l}\text { MC } \\
\text { Ajust. }\end{array}$ & $\begin{array}{l}\text { Valor } \\
\mathrm{F}\end{array}$ & $\begin{array}{l}\text { Valor } \\
\mathrm{p}\end{array}$ \\
\hline Factor & 1 & 0.013207 & 0.013207 & 60.42 & 0.0000 \\
\hline Error & 22 & 0.004809 & 0.000219 & & \\
\hline Total & 23 & 0.018016 & & & \\
\hline
\end{tabular}

Tabla 4 Anova de productividad aplicado a la línea 3 Fuente: Recuperado de Minitab 17
Para este análisis el valor de $\mathrm{p}$ es igual a 0 , lo que nos indica que rechazamos Ho y aceptamos Ha. Por lo tanto concluimos que si hay diferencia significativa entre tener o no TPM para la variable de productividad.

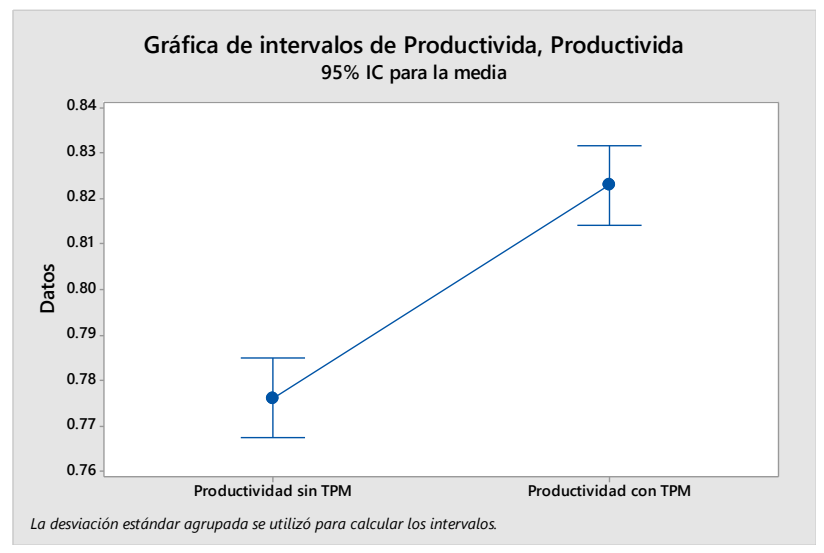

Gráfica 1 Intervalos de medias para la productividad aplicada a la línea 3

Fuente: Recuperado de Minitab 17

También se probaron los supuestos que se deben cumplir para poder realizar un diseño de experimentos completamente al azar los cuales se muestran en los anexos correspondientes.

Desperdicio.

Hipótesis a probar.

Ho: $\mu_{\operatorname{sinTPM}}=\mu_{\text {conTPM }}$

Ha: $\mu_{\operatorname{sinTPM}} \neq \mu_{\text {conTPM }}$

Para la parte de desperdicio se calculó para los 12 meses del año.

\begin{tabular}{|c|c|}
\hline $\begin{array}{c}\text { Desperdicio sin } \\
\text { TPM }\end{array}$ & $\begin{array}{c}\text { Desperdicio con } \\
\text { TPM }\end{array}$ \\
\hline 0.019 & 0.007 \\
\hline 0.025 & 0.007 \\
\hline 0.024 & 0.005 \\
\hline 0.042 & 0.005 \\
\hline 0.038 & 0.006 \\
\hline 0.039 & 0.006 \\
\hline 0.022 & 0.007 \\
\hline 0.022 & 0.006 \\
\hline 0.038 & 0.005 \\
\hline 0.019 & 0.005 \\
\hline 0.018 & 0.005 \\
\hline 0.024 & 0.005 \\
\hline
\end{tabular}

Tabla 5 Resultados de desperdicio sin y con TPM Fuente: Elaboración propia

Usando el software de minitab 17 se obtuvo el siguiente análisis de varianza, con un alfa de 0.05 .

LANDEROS-CORREA, Carmen, CHIHUAQUE-ALCANTAR, Jesús, MELESIO-MORENO, Ma. Guadalupe y GALVÁN-GARCÍA, María Isabel. Análisis de los factores de productividad, desperdicio y confiabilidad de los equipos, al implementar TPM en una empresa del sector automotriz. Revista de Ingeniería Industrial. 2019 


\begin{tabular}{|c|c|c|c|c|c|}
\hline Fuente & GL & $\begin{array}{l}\text { SC } \\
\text { Ajust. }\end{array}$ & $\begin{array}{l}\text { MC } \\
\text { Ajust. }\end{array}$ & $\begin{array}{l}\text { Valor } \\
\mathrm{F}\end{array}$ & $\begin{array}{l}\text { Valor } \\
\text { p }\end{array}$ \\
\hline Factor & 1 & 0.002836 & 0.002836 & 69.15 & 0.0000 \\
\hline Error & 22 & 0.000902 & 0.000041 & & \\
\hline Total & 23 & 0.003739 & & & \\
\hline
\end{tabular}

Tabla 6 Anova de desperdicio aplicado a la línea 3 Fuente: Recuperado de Minitab 17

Para este análisis el valor de p es igual a 0 , lo que indica que se rechaza. Hoy se acepta Ha. Por lo tanto, se concluye que si existe diferencia significativa entre tener o no TPM para la variable de desperdicio.

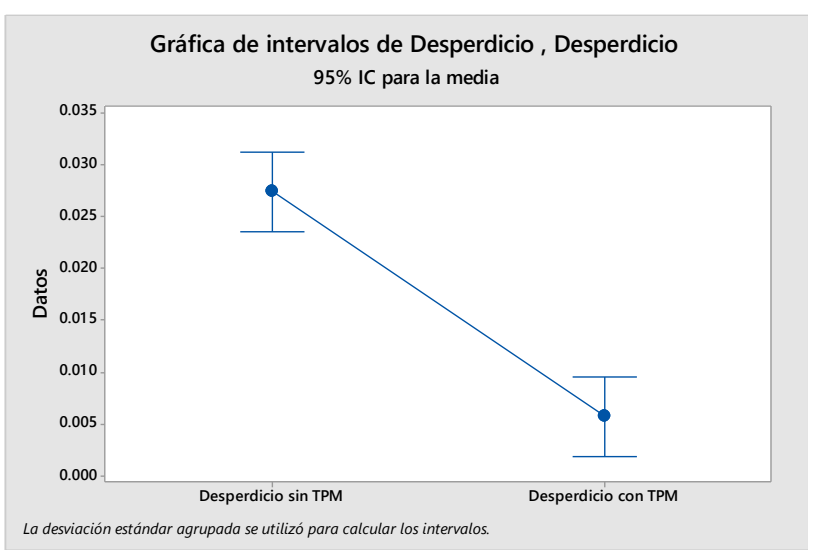

Gráfica 2 Intervalos de medias para el desperdicio aplicado a la línea 3

Fuente: Recuperado de Minitab 17

\section{Confiabilidad}

Hipótesis a probar.

Ho: $\mu_{\operatorname{sinTPM}}=\mu_{\text {conTPM }}$

Ha: $\mu_{\operatorname{sinTPM}} \neq \mu_{\text {conTPM }}$

Para la parte de confiabilidad se calculó para los 12 meses del año.

\begin{tabular}{|c|c|}
\hline $\begin{array}{c}\text { Confiabilidad } \\
\text { con TPM }\end{array}$ & $\begin{array}{c}\text { Confiabilidad } \\
\text { sin TPM }\end{array}$ \\
\hline 100.380 & 586.500 \\
\hline 178.410 & 201.610 \\
\hline 172.110 & 275.920 \\
\hline 125.380 & 471.000 \\
\hline 706.300 & 117.450 \\
\hline 255.210 & 599.580 \\
\hline 137.250 & 249.130 \\
\hline 198.040 & 189.500 \\
\hline 140.000 & 278.000 \\
\hline 149.000 & 296.000 \\
\hline 167.920 & 365.000 \\
\hline 232.590 & 265.000 \\
\hline
\end{tabular}

Tabla 7 Resultados de confiabilidad sin y con TPM Fuente: Elaboración propia
Usando el software de Minitab 17 se obtuvo el siguiente análisis de varianza, con un alfa de 0.05 .

\begin{tabular}{|c|c|c|c|c|c|}
\hline Fuente & GL & $\begin{array}{l}\text { SC } \\
\text { Ajust. }\end{array}$ & $\begin{array}{l}\text { MC } \\
\text { Ajust. }\end{array}$ & $\begin{array}{l}\text { Valor } \\
\text { F }\end{array}$ & $\begin{array}{l}\text { Valor } \\
\mathrm{p}\end{array}$ \\
\hline Factor & 1 & 73937 & 73937 & 2.99 & 0.098 \\
\hline Error & 22 & 544322 & 24742 & & \\
\hline Total & 23 & 618259 & & & \\
\hline
\end{tabular}

Tabla 8 Anova de confiabilidad aplicado a la línea 3 Fuente: Recuperado de Minitab 17

Para este análisis el valor de $\mathrm{p}$ es mayor a 0.05 , lo que indica que Ho es aceptada y que se rechaza $\mathrm{Ha}$, por lo tanto se concluye que no hay diferencia significativa entre aplicar o no TPM para este indicador.

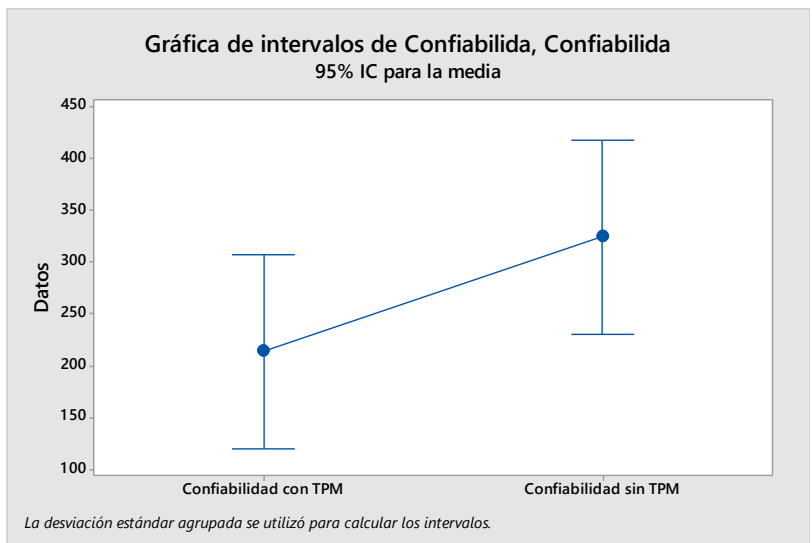

Gráfica 3 Intervalos de medias para la confiabilidad aplicada a la línea 3

Fuente: Recuperado de Minitab 17

Aunque gráficamente vemos una diferencia de medias, él análisis dice que ésta diferencia no necesariamente es por aplicar TPM.

\section{Conclusiones}

Esto solo aplica para esta línea en particular, si quisiéramos hacer una inferencia más general deberíamos hacer más análisis en otras líneas y en otras fábricas para poder sacar un comparativo y poder concluir algo más general.

Al aplicar el TPM y medir el indicador de productividad, se encontró como resultado de su anova, que si hay diferencia significativa en aplicar o no TPM, ya que nos mostró que el resultado que si hay diferencia en las medias.

Este análisis si cumple con los supuestos del Diseño de Experimentos, por lo cual aplicar o no TPM tiene un efecto en este indicador.

LANDEROS-CORREA, Carmen, CHIHUAQUE-ALCANTAR, Jesús, MELESIO-MORENO, Ma. Guadalupe y GALVÁN-GARCÍA, María Isabel. Análisis de los factores de productividad, desperdicio y confiabilidad de los equipos, al implementar TPM en una empresa del sector automotriz. Revista de Ingeniería Industrial. 2019 
El que el efecto sea positivo o negativo, dependerá de los objetivos de la empresa. Con el análisis de desperdicio que se realizó, se pudo comprobar mediante el ANOVA, que el TPM sí representa una diferencia significativa en este indicador. Es importante señalar que, en éste caso, también se cumplieron los supuestos del Diseño de Experimentos.

Por último, y con respecto a la confiabilidad; la primera comprobación consistió en verificar que los datos fueran normales. Sin embargo, cuando se aplica el TPM, los datos ya no se comportan de manera normal, por lo que al realizar el ANOVA; éste arroja como resultado que no existen diferencias entre aplicar o no TPM para este indicador. No obstante, al no cumplirse con uno de los supuestos del experimento no se puede concluir que el supuesto anterior, se actualice.

\section{Recomendaciones}

Como primer punto y en relación al análisis de confiabilidad, se recomienda es normalizar los datos, para verificar sí la aplicación del TPM puede o no ayudar a mejorar este indicador.

Una vez que los datos sean normales y cumplan con los demás supuestos, proceder a realizar el análisis de varianza y consecuentemente, validar las hipótesis de investigación.

\section{Referencias}

Box, G.E.; Hunter, J.S.; Hunter, W.G. (2008). Estadística para investigadores. Diseño, innovación y descubrimiento. Segunda Edición, Ed. Reverté, Barcelona.

Calvo, J. Lago, V. (Junio, 2004). Importancia del mantenimiento productivo total en la automatización de procesos. Recuperado el día 29 de Enero de 2019 de http://www.tecnicaindustrial.es/TIAdmin/Nume $\operatorname{ros} / 12 / 40 / a 40 . p d f$

García, J. Romero, J. Noriega, A. (14 de Marzo del 2011) El éxito del mantenimiento productivo total y su relación con los factores administrativos. Recuperado el día 9 de Diciembre de 2018 de http://www.scielo.org.mx/scielo.php?script=sci _arttext\&pid=S0186-10422012000400009
Gonzales Fernandez, F. J. (s.f.). Auditoria de Mantenimiento e Indicadores de Gestion. Principe Vergara. Obtenido de https://books.google.com.mx/books?id=o0cH7Nwk m3YC\&pg $=$ PA54\&lpg $=$ PA54\&dq $=m t t f+m t b f+m t t r$ + con + autor $\&$ source $=$ bl\&ots $=\mathrm{j} v j \mathrm{xN}-0 \mathrm{j} 52 \& \operatorname{sig}=2 \mathrm{CW}$ 09CPEh_Cnt_pA0ApYuMsRrLQ\&hl=es\&sa=X\&v ed=0ahUKEwi_2ISpotjNAhUD4oMKHf51AIsQ6A EIWjAM\#v $=$ onepage $\& \mathrm{q}=\mathrm{mttf} \% 20 \mathrm{mtbf} \% 20 \mathrm{mttr} \% 2$ 0con\%20autor\&f

Gutiérrez, H.; de la Vara, R. (2003). Análisis y diseño de experimentos. McGraw-Hill, México. Gutiérrez, H.; de la Vara, R. (2010). Calidad y productividad. McGraw-Hill, México

Huerta, F. (17 de 04 de 2015). am Leòn.Recuperado el día 28 mayo 2019 de http://www.am.com.mx/m/ valoragregado/guanajuato-lider-automotriz-195310. html

Huerta, Rosendo. (2006) Proceso de Análisis Integral de Disponibilidad y Confiabilidad como Soporte para el Mejoramiento Continuo de las Empresas. Reliability World 2006, junio 5 al 9 de 2006, Monterrey, México.

INEGI, AMIA. (12 de Diciembre, 2018). Conociendo la industria automotriz. Recuperado el día 13 de abril de 2019 de https://www.inegi.org.mx/contenidos/saladepre nsa/boletines/2018/OtrTemEcon/CndoIndAuto. pdf

Kurosawa, K. (1983). Medicion y Analisis de la Productividad a Nivel de Empresa. Recuperado el día 16 de abril 2019 de http://www.adingor.es/congresos/web/uploads/c io/cio2012/SP_02_Gestion_de_Operaciones_y_ Produccion/847-854.pdf

Partida, A. (06 de 11 de 2012). Mantenimiento \& Mentoring Industrial. Recuperado el 10 de Abril de http://mantenimiento-mi.es/2012/la-mportancia-deltpm-mantenimiento-productivo-total

Meyer, F.E., \& Stephens, M. P. (2006). Diseño de Instalaciones de Manufactura y Manejo de Materiales. Pearson, Prentice Hall.

Napolitano H. (sf) Diseño de Experimentos. Recuperado el día 3 de marzo de 2019 de https://s3.amazonaws.com/academia.edu.docum ents/46255110/disenos_fases.pdf?responsecontentdisposition=inline $\% 3 \mathrm{~B} \% 20$ filename $\% 3$ DDisenos_fases.pdf 
Olofsson, O. (2013). Eficiencia Global de los Equipos. Recuperado el 25 de mayo de 2019 de https://world-class-manufacturing.com/es/OEE/ oee-alculation.html

Pinto, Lucia (Diciembre, 2010). Mantenimiento productivo total. Recuperado el día 14 de abril 2019

de http://www.emb.cl/electroindustria/articulo.mvc ?xid $=1520$

Tanco M; Viles E; Pozueta L. (2009). Diferentes enfoques del diseño de experimentos (DOE). Recuperado el día 3 de marzo de 2019 de https://www.researchgate.net/profile/Martin_Ta nco/publication/43768706_Diferentes_enfoques _del_diseno_de_experimentos_DOE/links/0dee c51bb19ec1d50a000000.pdf

Tejada Gamarra, J. N. (2019). Propuesta de modelo de optimización de la disponibilidad de maquinaria y equipo del área de Maestranza de la empresa FAMAI, utilizando la metodología del Mantenimiento Productivo Total-TPM

Yepes, V. (Marzo del 2013) Diseño completamente al azar y ANOVA. Recuperado el día 4 abril de 2019 de https://victoryepes.blogs.upv.es/2013/04/27/dis eno-completamente-al-azar-y-anova/

\section{Anexos}

A continuación, se realizarán los cálculos de normalidad y de varianza para cada uno de los indicadores.

\section{Cálculo de Normalidad para Productividad}

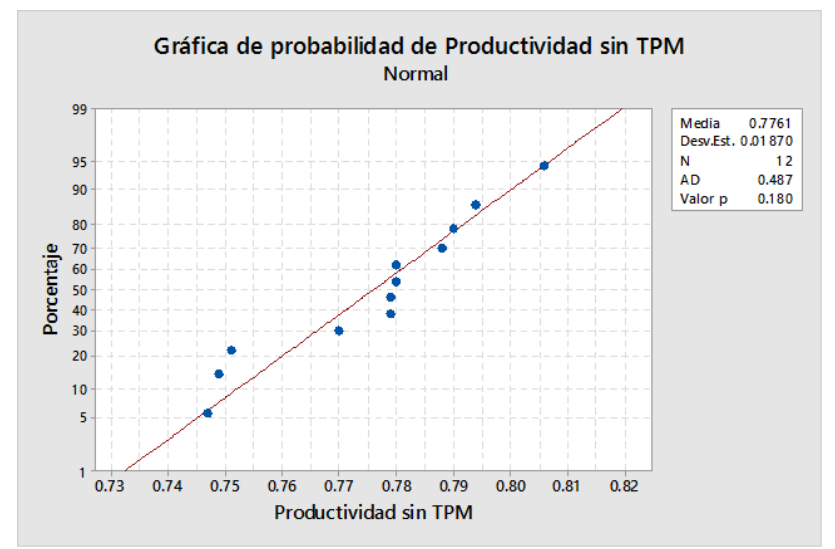

Gráfica 4 Gráfica de Normalidad aplicada a la productividad sin TPM

Fuente: Recuperado de Minitab 17

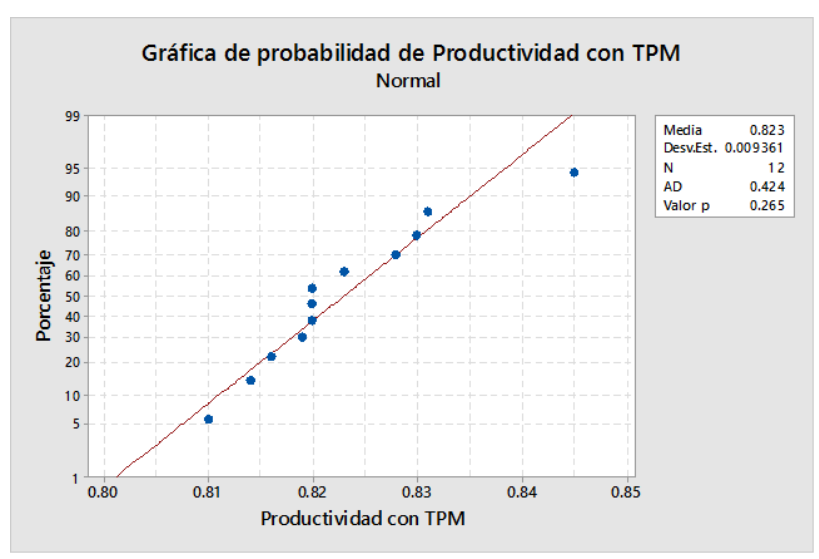

Gráfica 5 Gráfica de Normalidad aplicada a la productividad con TPM

Fuente: Recuperado de Minitab 17

Para la productividad ambos datos cumplen con la normalidad, debido a que valor $\mathrm{p}$ es mayor a 0.05 .

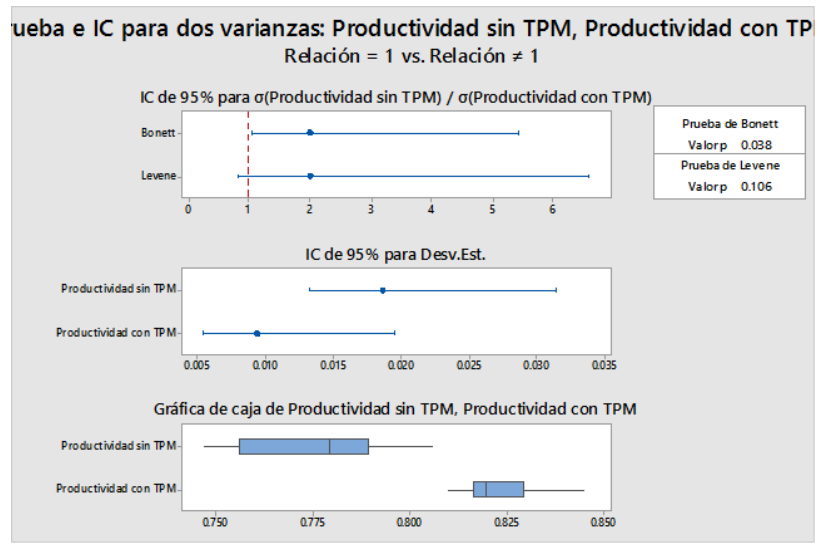

Gráfica 6 Prueba de Varianzas independientes aplicada a la productividad

Fuente: Recuperado de Minitab 17

Para la productividad las varianzas son diferentes, debido a que valor $\mathrm{p}$ es menor a 0.05 .

\section{Calculo de normalidad para el desperdicio}

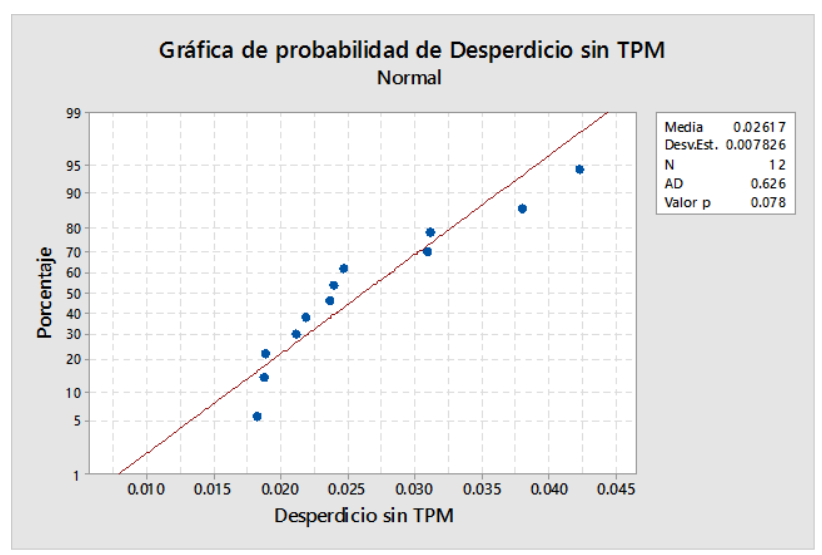

Gráfica 7 Gráfica de Normalidad aplicada al desperdicio sin TPM

Fuente: Recuperado de Minitab 17 


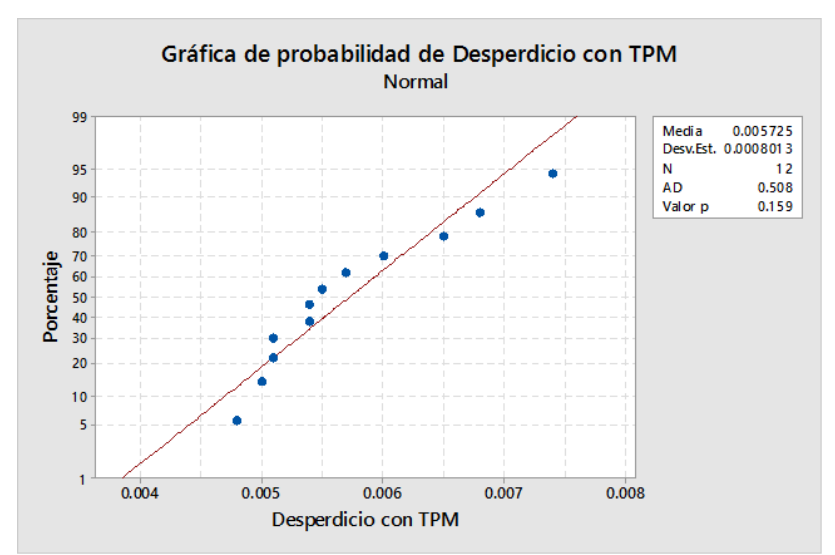

Gráfica 8 Gráfica de Normalidad aplicada al desperdicio con TPM

Fuente: Recuperado de Minitab 17

Para el desperdicio ambos datos cumplen con la normalidad, debido a que valor $\mathrm{p}$ es mayor a 0.05 .

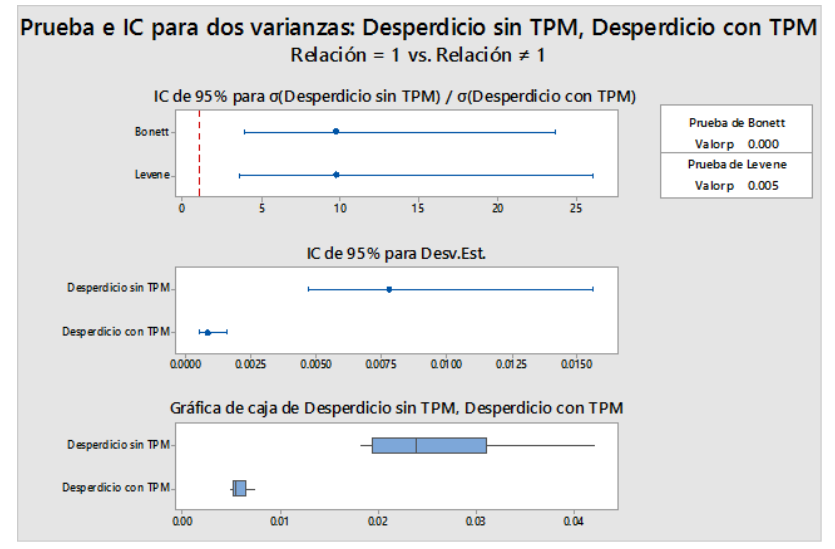

Gráfica 9 Prueba de Varianzas independientes aplicada al desperdicio

Fuente: Recuperado de Minitab 17

Para el desperdicio las varianzas son diferentes, debido a que valor p es menor a 0.05 .

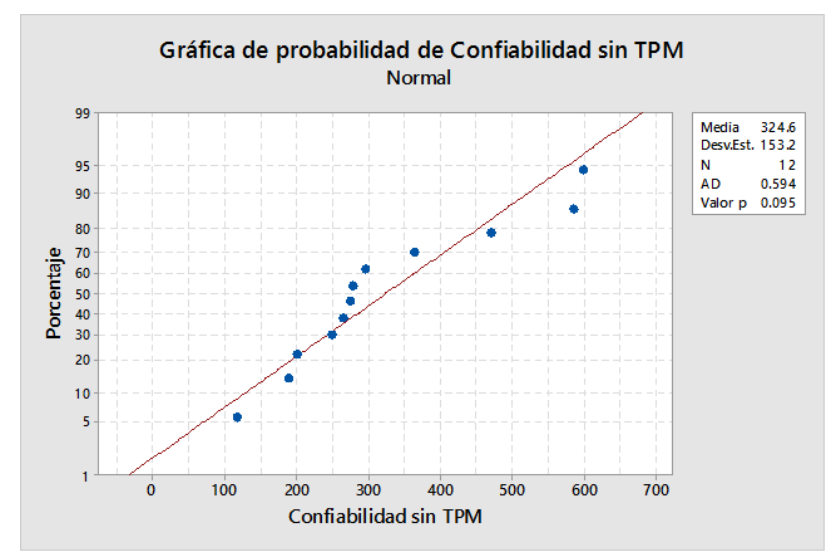

Gráfica 10 Gráfica de Normalidad aplicada a la confiabilidad sin TPM

Fuente: Recuperado de Minitab 17

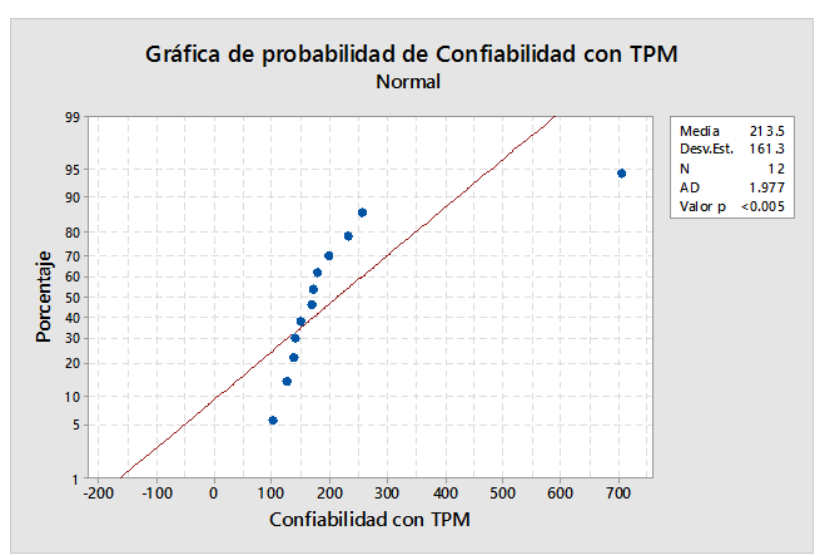

Gráfica 11 Gráfica de Normalidad aplicada a la confiabilidad con TPM

Fuente: Recuperado de Minitab 17

Para la confiabilidad sin TPM si cumple con el supuesto de normalidad, pero cuando aplicamos el TPM los datos ya no se comportan de manera normal.

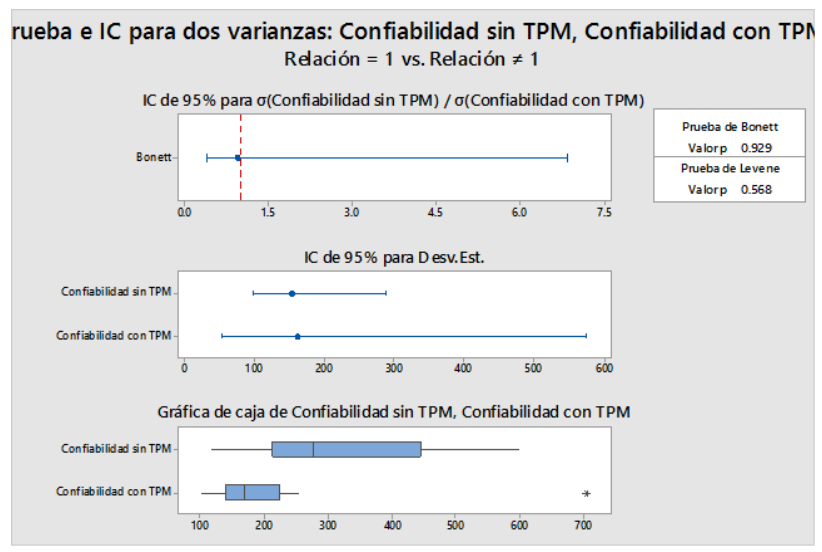

Gráfica 12 Prueba de Varianzas independientes aplicada la confiabilidad

Fuente: Recuperado de Minitab 17

Para el desperdicio las varianzas son iguales, debido a que valor p es mayor a 0.05 . Por lo cual el ANOVA no se puede aplicar con este tipo de datos. 